Sociologie et sociétés

\title{
Les identités sexuelles et les travailleuses de l'industrie du sexe à l'aube du nouveau millénaire
}

\section{Colette Parent}

Volume 33, numéro 1, printemps 2001

Les formes de la pénalité contemporaine : Enjeux sociaux et politiques

URI : https://id.erudit.org/iderudit/001544ar

DOI : https://doi.org/10.7202/001544ar

Aller au sommaire du numéro

Éditeur(s)

Les Presses de l'Université de Montréal

ISSN

0038-030X (imprimé)

1492-1375 (numérique)

Découvrir la revue

Citer cet article

Parent, C. (2001). Les identités sexuelles et les travailleuses de l'industrie du sexe à l'aube du nouveau millénaire. Sociologie et sociétés, 33(1), 159-178.

https://doi.org/10.7202/001544ar d'utilisation que vous pouvez consulter en ligne. 


\section{Les identités sexuelles et les travailleuses de l'industrie du sexe à l'aube du nouveau millénaire}

COLETTE PARENT

Département de criminologie

Université d'Ottawa

25, Université

Ottawa (Ontario), Canada $\mathrm{K}_{1} \mathrm{~N} 6 \mathrm{~N}_{5}$

Courriel : coparent@uottawa.ca

$\mathrm{D}$ ANS NOS SOCIÉTÉS OCCIDENTALES, selon Foucault, le sexe nous révèle à nousmêmes notre vérité et nous permet de lire celle des autres. Tout notre être, toute notre individualité, toute notre histoire de vie peut être scrutée et comprise à partir de la concupiscence et du désir (Foucault, 1976). Ce dispositif de sexualité qui s'est mis progressivement en place à partir XVII ${ }^{\mathrm{e}}$ siècle a permis la constitution d'une science sur la sexualité sous l'autorité d'un contingent de spécialistes; parmi eux, des médecins, des psychologues, des travailleurs sociaux, des criminologues. On note alors l'émergence de tout un discours sur les sexualités polymorphes, les unes «normales» et les autres «perverses». Attachées à ces sexualités, on retrouve des identités : celle de la femme, de l'enfant, de l'homosexuel, de la prostituée, etc. Le sexe en Occident à partir de cette époque se voit donc soumis à une double démarche de connaissance : d'un côté le développement de tout un savoir "scientifique», de l'autre celui d'un savoir réflexif qui nous amène à chercher en chacun de nous notre identité, révélée par le sexe. En effet, comme le souligne Foucault (1976, p. 102), cette orientation nous a fait passer

${ }^{*}$ Nous tenons à remercier un des évaluateurs de cet article pour ses précieux commentaires critiques qui nous ont aidé à préciser les objectifs et les limites méthodologiques de notre étude aussi bien qu'à y apporter un certain nombre de corrections et d'éclaircissements. 
en quelques siècles "presque tout entier - nous, notre corps, notre âme, notre individualité, notre histoire - sous le signe de la concupiscence et du désir. Dès qu'il s'agit de savoir qui nous sommes, c'est elle qui nous sert de clef universelle». On y retrouve, d'un côté, le monde de la perversion; les identités sexuelles de l'homosexuel et de la prostituée y prennent place. Celles-ci contribuent à consolider en contrepartie le sexe «naturel», «normal» comme mode de spécification des individus. Les femmes, en tant que catégorie de sexe, n’y échappent pas. Si leur identité est solidement construite comme une catégorie naturelle de genre, leur sexualité n'en est pas moins partie prenante essentielle de leur identité femme. Tant le critère de l'orientation sexuelle que celui du choix des pratiques sexuelles et de la subjectivité dont elles sont investies contribuent à construire l'identité femme «normale» et circonscrivent les possibilités de définition et de choix sexuel des femmes. Si on peut dire que l'identité sexuelle des homosexuels et des prostituées absorbe toute leur individualité et entraîne la stigmatisation, l'identité sexuelle «normative» des femmes constitue une dimension incontournable de l'oppression dont elles sont l'objet.

Comme on peut le constater par les remarques précédentes de Foucault, il est difficile de réduire ses propos exclusivement à la sexualité dite "perverse»; ils portent aussi sur la spécification de la sexualité considérée normale et montrent le lien entre cette normalisation et l'identité de la personne ou du groupe. De toute façon, quoi qu'il en soit du point de vue particulier de Foucault, son analyse nous fournit assez d'éléments pour proposer une approche reliant l'identité femme et la régulation de leur sexualité.

Mais comme les résistances constituent «l'irréductible vis-à-vis» (Foucault, 1976, p. 127) des relations de pouvoir, on peut observer depuis le XIX ${ }^{e}$ siècle la lutte de plusieurs groupes contre cette vaste entreprise de définition d'eux-mêmes et de leur identité. La «révolution sexuelle» des années 1960 en Occident constitue d'ailleurs un moment pivot de cette résistance. Différents groupes sociaux, groupes de femmes, de gais et de lesbiennes aussi bien que de prostituées, ont alors fait entendre leur voix sur la place publique. Chacun à leur façon, ils ont exprimé leur révolte contre les identités de groupe dans lesquels ils se retrouvaient enfermés et qui déterminaient en quelque sorte la position sociale qu'ils pouvaient occuper (Weeks, 1995, p.103). En fait, tant les femmes, les homosexuels et les lesbiennes que les prostituées ont non seulement rejeté les étiquettes qu'on leur avait longtemps imposées, mais ont cherché à établir pour chacun et chacune d'entre eux un espace ouvert et socialement légitime pour découvrir ou vivre autrement leur sexualité. Cette révolution sexuelle des années 1960 n’a pas fait table rase du dispositif de sexualité, on le sait maintenant; elle a plutôt constitué une «révolution non achevée» (Weeks, 1993a). Inachevée certes et qui, à première vue, a produit des effets différentiels selon les groupes. Dans le cadre de cet article, nous nous interrogeons principalement sur le parcours des regroupements des prostituées. Comment expliquer ce que plusieurs ont défini comme l'échec du mouvement? Pour éclairer notre questionnement, nous utilisons en contre-exemple et présentons, à grands traits, quelques éléments marquants du parcours de résistance et 
de lutte des femmes et des gais et lesbiennes. Écartons néanmoins tout malentendu : il ne s'agit pas de refaire l'histoire de ces mouvements ni de rendre compte de leur complexité interne, mais tout simplement de baliser certains points de comparaison susceptibles de jeter un éclairage pertinent sur notre sujet : le destin incertain de la lutte des prostituées.

Nous nous heurtons cependant aussi à un problème d'ordre méthodologique : en effet, quelle procédure convient-il d'adopter pour approcher notre sujet? Pour résoudre ce problème, disons que nous n'essayons pas ici de décrire une lutte, celle des prostituées, et moins encore l'histoire d'un ou plusieurs mouvements sociaux à la fois. Outre les limites d'espace dans le cadre d'un article, ceci réclamerait des procédures méthodologiques particulières, comme le choix d'un contexte géographique spécifique, d'une période, etc. Nous analysons plutôt ici un problème qui peut être circonscrit par les questions suivantes : pourquoi les prostituées n'ont-elles pas réussi à transformer une attente normative à l'égard de leurs pratiques sexuelles — soutenue, qui plus est, par le caractère contraignant d'une loi pénale - en une pratique permissive et juridiquement régulée comme une activité professionnelle? Comment se fait-il qu'elles n’ont même pas réussi à obtenir la décriminalisation de leurs activités sexuelles? Comment expliquer que les femmes et les gais et les lesbiennes aient réussi à introduire, du moins semble-t-il, certaines conditions de possibilité de changements au niveau de leur identité sexuelle et non pas les prostituées? On voit bien, nous l'espérons, sur quel point la comparaison avec les autres mouvements est appelée à intervenir dans le cadre de nos propos. Nous allons aussi insister sur les changements juridiques considérant que le droit a joué et joue un rôle central au niveau des enjeux autour de la définition des identités sexuelles. Certes, il ne les détermine pas, mais il généralise, stabilise et honore certaines attentes normatives de comportement, stigmatise les comportements et les personnes qu'il prend en charge, impose à l'occasion des sanctions afflictives et fournit aussi, par conséquent, les conditions de possibilités pour le développement des pratiques de résistance. Cet angle d'analyse nous permet d'examiner a contrario la barrière juridique à laquelle se bute encore et toujours le mouvement des prostituées.

Enfin, comme l'écrit Foucault (1980) dans son texte sur La poussière et le nuage, les deux types de démarches ne peuvent pas se construire à partir des mêmes critères méthodologiques. La recherche qui part de problèmes cherche à marquer les «temps forts" de l'analyse (Foucault, 1980, p. 30); par conséquent, nous devons renoncer à ce que nous fait apprendre la richesse (infinie) du détail. C'est la règle de la pertinence qui compte pour nous, et non celle de l'exhaustivité ou de la diversité interne du thème en lui-même.

\section{LA MISE EN PLACE DES IDENTITÉS SEXUELLES AU XIX ${ }^{\mathrm{e}}$ SIÈCLE}

Dans ce processus de déconstruction et re-définition du sexe en Occident, la famille devient un site privilégié. Au cours du XIX ${ }^{\mathrm{e}}$ siècle, le mariage gagne une légitimité élargie, du moins parmi les classes dominantes, et devient synonyme de respectabilité et de stabilité (Weeks, 1981, p. 24). Pour la première fois dans l'histoire de l'Occident, la struc- 
ture de la famille est associée à l'amour, à l'intimité. Elle est alors appelée à jouer un rôle important dans la surveillance et le contrôle des comportements sexuels; elle trace la frontière entre le sexe licite et illicite (Weeks, 1981, p. 24-25).

Parmi les sexualités qui font l'objet de réflexions, notons d'abord celle des femmes. Le nouvel idéal domestique qui émerge avec le pouvoir bourgeois les cantonne dans des rôles sociaux d'épouses et de mères. Leur sexualité est définie comme secondaire, circonscrite par leurs instincts maternels. On conçoit d'ailleurs que ce sont ces mêmes instincts maternels qui les amènent à se soumettre à la passion sexuelle des hommes ${ }^{1}$ (Weeks, 1981, p. 41). Pour le reste, la sexualité des femmes ne s'inscrit pas sous le signe du plaisir mais du danger : danger de maladies vénériennes, de grossesses, de perte de réputation (Dubois et Gordon, 1983). Durant la même période, la masculinité est de plus en plus associée à la force physique et à la volonté et l'on dénonce la masturbation comme dépravante et cause de l'homosexualité (Weeks, 1981, p. 40).

C'est dans ce contexte que se forme au fil du XIX ${ }^{e}$ siècle l'identité sexuelle marginale de l'homosexuel ${ }^{2}$. Il se dresse comme un personnage défini tout entier à partir de ses pratiques sexuelles, qui ne constituent plus une sorte de péché mais le reflet de sa nature. On lui prête une enfance, une vie, un caractère, une morphologie, une anatomie, etc., qui renvoient à sa sexualité. Tout en lui l'évoque, il se profile comme «un hermaphrodite de l'âme» (Foucault, 1976, p. 59). Les lois et leur application aussi bien que la médecine et les sciences sociales ont largement influencé cette nouvelle conception des pratiques homosexuelles. Notons, par exemple, que jusqu'au milieu du Xvi ${ }^{\mathrm{e}}$ siècle en Angleterre, la sodomie relevait des tribunaux ecclésiastiques (Kinsman, 1996, p. 49); comme tout autre pratique sexuelle non associée à la procréation, celle-ci tombait dans la catégorie des péchés. L'infraction de sodomie ne sera créée par le Parlement britannique qu'en 1533. À la fin du XIx ${ }^{e}$ siècle, on inclut de nouvelles lois qui distinguent la bestialité de l'homosexualité et qui condamnent non seulement la sodomie et la tentative de sodomie mais tout acte sexuel posé entre hommes (Weeks, 1981, p. 102). Ces lois ne sont pas appliquées très systématiquement mais lorsqu'on intervient, la rigueur est de mise. En 1890, le Canada emboîte le pas et adopte « une loi qui vise à punir tout acte de grossière indécence commis en public ou en privé entre adultes consentants de sexe masculin» (Demers, 1984, p. 791). Dans la même foulée, on commence à offrir des explications médicales pour cette conduite : l'homosexuel est classé comme une catégorie de dégénérés. Dans la communauté scientifique, en Europe comme en Amérique du Nord, on se demande si cette déficience est innée ou acquise, profondément ancrée dans l'individu ou traitable, socialement tolérable ou pas (Weeks, 1981, p. 104). Notons que dans le cadre des campagnes de réforme morale à la fin du XIX

1. Ne nous méprenons pas. Comme l'indique weeks (1981, p. 30), des intérêts économiques sont intégrés dans ces préoccupations. La chasteté des femmes assure que les enfants même du mari hériteront des biens, que les fils pourront devenir des associés loyaux et qu'à leur tour, en se mariant, les filles contribueront aux alliances d'affaires.

2. Le terme lui-même daterait du Xıx ${ }^{e}$ siècle. Il aurait été inventé en 1868 par un médecin autrichien nommé Karoly Maria Kertbeny. Il aurait ensuite été repris dans les ouvrages médicaux. Voir Tamagne (1998, p. 48). 
siècle, on se soulève contre le désir masculin débridé, qu'il prenne la forme de pratiques homosexuelles ou de recherche de services sexuels en dehors des liens du mariage. On relie les conduites homosexuelles à celles des hommes qui séduisent et corrompent les jeunes filles (Weeks, 1981, p. 106). Il semble bien que des hommes de classe dominante, limités dans leurs activités sexuelles homosexuelles par le code moral rigide imposé à leur classe, se soient tournés vers de jeunes hommes de classe ouvrière qu'ils ont définis comme étant moins hostiles aux relations entre hommes (Kinsman, 1996, p. 57). Dans le cadre de ces relations, illégales et largement furtives, l'offre d'argent entre régulièrement en jeu de sorte que la prostitution fait nécessairement partie du monde homosexuel (Weeks, 1981, p. 112-14). Par contre, les relations sexuelles entre femmes sont exclues des dispositions criminelles britanniques et canadiennes adoptées durant cette période et l'identité lesbienne s'est constituée beaucoup plus lentement ${ }^{3}$.

À la même époque, la prostituée joint les rangs de ce "petit peuple» marqué du sceau de la perversion. Jusque-là, les pratiques de prostitution faisaient partie du destin des femmes de classe ouvrière en ce qu'elles leur offraient, à certains moments de leur vie, un moyen de subsistance (le seul parfois) pour elles-mêmes et leurs enfants (Walkowitz, 1980). À partir du XIx siècle, la prostitution prend figure de véritable fléau social, la prostituée est définie comme une paria (Parent et Coderre, 2000; Walkowitz, 1980, 1991). On la tient responsable de la propagation des maladies vénériennes et l'on craint non seulement pour la santé des clients et de leur famille innocente mais également pour celle des générations futures et de la nation toute entière. On dénonce son influence morale qui peut se révéler d'autant plus importante et pernicieuse qu'elle fait partie de la classe ouvrière et risque de corrompre ceux et celles qui mènent des vies vertueuses. Si l'on reconnaît dans les rangs des prostituées des jeunes filles écrasées par la misère, on les dépeint également comme des femmes dépravées. Peu à peu, la prostituée devient l'antithèse de la femme vertueuse, «normale». L'adoption de lois qui resserrent les contrôles sur la prostitution tant en Europe qu'en Amérique du Nord à la fin du $\mathrm{XIX}^{\mathrm{e}}$ siècle accompagne et enracine ces changements. Au Canada, entre 1867 et 1895, les législateurs adoptent une série de lois visant à éliminer toutes les activités associées à la prostitution (Conseil consultatif canadien sur la situation de la femme, 1984, p.12-15) ${ }^{4}$.

3. Au XIX ${ }^{e}$ siècle, le travestisme et les amitiés romantiques ont fourni des canaux d'exploration de la sexualité homosexuelle pour les femmes mais on trouve peu de traces de sous-culture travestie ou lesbienne durant ce siècle sauf à Paris (Walkowitz, 1991, p. 413). Au début du xx siècle, on note l'émergence d'une culture lesbienne tant parmi la classe ouvrière que moyenne en Europe et en Amérique du Nord. Schulz (1998) note que si le droit s'est montré plutôt silencieux sur les relations sexuelles entre femmes, la volonté des femmes de modifier leur statut sexuel à travers le travestissement a été largement réprimée. Cette initiative était passible de mort en France au Xvi ${ }^{\text {e }}$ siècle. Elle affirme par ailleurs que «l'apparente neutralité du droit cache un véritable déni de la sexualité féminine en général, et de l'homosexualité féminine en particulier, et non, comme on pourrait le penser, une plus grande tolérance» (Schulz, 1998, p. 114-115). Au Canada, la loi contre les actes de grossière indécence entre adultes ne touchera les femmes que lors de la révision du Code criminel de 1953-54 (Demers, 1984, p. 791).

4. Au fil des ans, les lois viseront les prostituées dans les rues comme dans les maisons de prostitution, les clients, les propriétaires et tenanciers de maisons de prostitution, les souteneurs ou encore ceux qui participent à ce commerce. Voir à ce sujet, Cellard et Pelletier (1998). 
Durant les années 1860, on impose presque partout en Europe un régime réglementariste : les prostituées doivent être fichées et se soumettre à des examens médicaux périodiques (Walkowitz, 1991, p. 396). Les explications scientifiques qui émergent de la médecine, de la psychologie, de la criminologie, etc., contribuent à consolider cette nouvelle identité sexuelle marginale. La criminologie traditionnelle, par exemple, définit la prostitution comme le crime par excellence des femmes et offre des explications basées sur les déficiences des prostituées : ces femmes souffrent d'un déficit biologique et/ou psychologique et/ou social en comparaison des femmes honnêtes, normales qui mènent des vies vertueuses et répondent à leur rôle naturel de mères et d'épouses ${ }^{5}$.

Mais là où l'on trouve rapports de pouvoir, on trouve également des points de résistance (Foucault, 1976, p. 126) qui varient selon les groupes. Rappelons d'abord que les femmes qui endossent l'identité prescrite pour les femmes en général échappent à la stigmatisation contrairement aux homosexuels et aux prostituées. Elles n'en sont pas moins fortement contraintes comme sujets sexuels et comme groupe sexué et décideront éventuellement de lutter pour leur libération. Cette lutte collective est par ailleurs plus tardive ${ }^{6}$. Par contre, très tôt, face aux lois, aux discours psychiatriques et juridiques sur l'homosexualité, à certaines poursuites judiciaires célèbres (dont celle contre Oscar Wilde en Angleterre en 1895) et à la panique morale déclenchée contre les relations homosexuelles à cette époque, certains hommes commencent à s'identifier comme des homosexuels. Ils prennent appui sur les langages psychiatrique et juridique mêmes pour réclamer la reconnaissance de la légitimité ou encore de la naturalité de l'homosexualité (Weeks, 1981). Les travailleuses dans le domaine des services sexuels ont également réagi contre la stigmatisation. Dès la fin du XIX ${ }^{e}$ siècle en Angleterre, elles empruntent le langage du droit pour défendre leur intégrité physique face aux contrôle médical et policier mis en place à cette époque (Walkowitz, 1991, p. 402) et organisent des charivaris en guise de protestation contre la répression dont elles font l'objet (Walkowitz, 1980, p. 27).

\section{DE LA RÉVOLUTION SEXUELLE}

Les années 1960 en Occident sont marquées par des changements significatifs au niveau de l'État capitaliste : l'État assume plus de responsabilités au niveau de la reproduction sociale et s'implique dans les politiques familiales; les femmes, mariées comme célibataires, maintiennent leur engagement sur le marché du travail et ont recours à la pilule et l'avortement pour contrôler les naissances; on assiste à une expansion du marché de consommation. Ce faisant, on note une érosion des régulations sexuelles et familiales de l'époque victorienne, «la production d'un imaginaire sexuel plus visible et l'émergence de cultures lesbienne et homosexuelle visibles» (Kinsman, 1996, p. 288). Les changements au niveau de la sexualité apparaîtront d'entrée de jeu fort révolutionnaires. En effet, au sexe associé à la reproduction, dans le cadre d'une relation

5. À ce sujet, voir Parent et Coderre (2000).

6. Notons que les luttes féministes du début $\mathrm{du} \mathrm{xx}^{\mathrm{e}}$ siècle ne porteront pas principalement sur l'oppression relative à la sexualité des femmes. 
hétérosexuelle monogame légitimée par le mariage, au sexe basé sur un double standard de moralité selon la catégorie de sexe, on a voulu opposer le sexe aux multiples significations : symbole amoureux certes mais aussi instrument de communication, source de plaisir. Cette ouverture impliquait la possibilité de relations sexuelles avant le mariage, de relations sexuelles sans liens amoureux, de cohabitation sans mariage, de relations sexuelles homosexuelles. Durant cette période, le mouvement féministe, le mouvement de libération des gais et lesbiennes, les regroupements de défense des droits de prostituées ont participé à cette tentative pour redéfinir le sexe en Occident. Ils ont cherché, chacun à leur façon, à faire éclater les marqueurs étroits de leur identité sexuelle et à se tailler un espace social ouvert et légitime. Quelles initiatives ont-ils mises de l'avant et quels impacts celles-ci ont-elles eus?

\section{Le mouvement de libération des femmes et la question de la liberté sexuelle}

Dans les premiers moments, on constate que les féministes ont appuyé la diversité sexuelle et la définition de la sexualité comme champ de plaisir, y voyant un espace de libération pour les femmes (Richardson, 1993, p. 86). Mais très tôt plusieurs d'entre elles ont mis en évidence qu'amour libre et libération sexuelle n'étaient pas synonymes; si les femmes pouvaient se libérer ainsi des normes strictes de chasteté, elles ne se libéraient pas pour autant du pouvoir masculin sur elles. La libération sexuelle risquait bien dans ce contexte de favoriser d'abord et avant tout les intérêts des hommes ${ }^{7}$. Les féministes radicales en particulier ${ }^{8}$ ont proposé que le fondement de l'oppression des femmes par les hommes reposait sur l'appropriation de la sexualité des femmes par ces mêmes hommes; pour se libérer, les femmes devaient pouvoir définir et contrôler leur propre sexualité et leur corps. Au fil des années, la lutte des femmes a porté sur le pouvoir de définir leur sexualité et de se poser comme des partenaires égales dans le champ des relations intimes, que ce soit entre hommes et femmes ou entre femmes. Elles ont mis en cause la morale victorienne qui associait la sexualité normale à la procréation et qui subordonnait la sexualité des femmes à celle-ci. Elles ont ainsi acquis la possiblité de se définir de façon diverse comme êtres sexués et de choisir des relations intimes conséquentes. La cellule familiale traditionnelle a éclaté pour laisser place à des modes de vie multiples et on dissocie maintenant beaucoup plus valeurs religieuses et valeurs sexuelles, «vérités scientifiques» et moralités des conduites sexuelles. La responsabilité des comportements sexuels, comme le remarque Weeks (1993a, p. 8), repose maintenant sur l'individu.

Pour les femmes par ailleurs, au-delà de ces changements, la sexualité demeure attachée à la sphère de l'intimité et fournit toujours les bases premières de la signification de soi et de son insertion dans le monde. Selon Giddens (1992, p. 50), la diversité

7. Notons ici que durant les années 1980, les féministes ont développé des positions diverses et plus ou moins opposées sur la sexualité mais la pensée féministe radicale réussira à dominer l'agenda féministe sur cette question. Voir à ce sujet, entre autres, Chapkis (1997), Richardson (1993), Seidman (1992).

8. Pour une présentation de la pensée féministe radicale, voir, entre autres, Descarries-Bélanger et Roy (1988), Jaggar (1983), Tong (1984). 
sexuelle n'a pas sonné le glas de l'amour romantique mais a entraîné une redéfinition de ses paramètres. En effet, si l'amour n'est plus associé à la virginité obligatoire des jeunes filles et aux fréquentations traditionnelles qui culminent avec une demande en mariage, il demeure un élément fondamental de la destinée que veulent se tailler les jeunes filles. "Contrairement à la plupart des hommes, la majorité des femmes continuent d'identifier l'entrée dans le monde adulte avec la formation de liens» (Giddens, 1992, p. 53). L'avenir en termes de carrière, d'insertion sur le marché du travail, n'a pas réussi à déloger la quête relationnelle dans les options de vie des femmes. Mais cette fois, on parle de relations plutôt que de mariage et le terme renvoie à des liens affectifs profonds et stables entre deux partenaires. Pour Giddens (1992, p. 58), ce phénomène de restructuration de l'intimité pour lequel il propose le terme de «relation pure», implique une relation librement choisie par les deux partenaires; ce choix se fait à partir de ce que chacun peut tirer de cette relation intime et perdure aussi longtemps qu'elle est satis-

faisante pour les deux. Pour les femmes en somme, le sexe participe toujours de la construction de soi, de la quête de sa destinée individuelle. Aussi, si on peut parler de changement au niveau de l'identité sexuelle des femmes, si on reconnaît qu'elles n'associent plus leur vie à une seule relation affective consacrée dans le mariage traditionnel, la plupart basent toujours leur vie sur une relation affective et sexuelle stable. On note bien le maintien de l'association entre identité personnelle et sexualité.

\section{Les gais et les lesbiennes dans l'espace public}

Le mouvement des années 1960 a également permis un certain nombre d'acquis pour les homosexuels. En 1954, en Angleterre, le Comité Wolfenden chargé d'enquêter sur le «sujet dégoûtant de l'homosexualité et de la prostitution» (Kinsman, 1996, p. 213) s'est donné un cadre d'analyse basé sur la distinction privé/public, ce qui l'a amené à considérer qu'un certain nombre de comportements relevaient davantage de la moralité privée que du contrôle public (Kinsman, 1996, p. 214). Cette catégorisation, appliquée à la sexualité, a eu pour effet de revoir ce qui ne relevait pas du système juridique. Il a alors recommandé la décriminalisation des rapports sexuels entre deux hommes en privé laissant le contrôle éventuel de ces activités, qu'il ne s'agissait aucunement de légitimer par le droit, aux savoirs psychiatriques, psychologiques, médicaux et à leurs institutions. Quant aux actes homosexuels en public, il a prôné une réaction vigoureuse et sévère. La désescalade pénale proposée par le Comité n'entrainera des effets juridiques en Angleterre et au pays de Galles qu'en 1967, suite à une série de campagnes menées par des sympathisants à la cause homosexuelle. La stratégie adoptée par le Comité Wolfenden influera sur les législations subséquentes en Angleterre et sur l'analyse canadienne concernant la régulation des activités sexuelles. Les sexualités « déviantes» devront échapper au regard public.

Au Canada, pendant les années 1950 et 1960, on note la formation de réseaux d'homosexuels et de lesbiennes qui favorisent l'émergence d'une culture spécifique homosexuelle et lesbienne et l'organisation de groupes de défense de leurs intérêts (Kinsman, 1996, p. 224). Plusieurs églises s'intéressent à la question, recommandent 
une attitude plus libérale face à l'homosexualité et appuient une réforme juridique. Durant les années 1960, les média commencent également à couvrir les questions qui touchent l'homosexualité et plusieurs groupes de professionnels, les juristes y compris, offrent pour des raisons diverses, leur support à la réforme juridique. En 1969, le Parlement canadien adopte le projet de loi 150, un bill omnibus qui touche non seulement à la question de l'homosexualité mais aussi à l'avortement, à la contraception, au contrôle des armes, etc. Le gouvernement s'appuie aussi sur la catégorisation privé/ public et sur les différences entre les domaines du droit et de la morale pour justifier sa volonté de décriminaliser l'homosexualité (Kinsman, 1996, p. 269). Mais comme les débats parlementaires en font foi, cette réforme juridique ne constitue pas aux yeux du gouvernement et de l'ensemble des parlementaires qui la supportait une légitimation de l'homosexualité : beaucoup d'entre eux considéraient toujours ces actes comme odieux mais étaient persuadés que l'homosexualité était une maladie et qu'on devait penser en termes de traitement plutôt que de punition. L'adoption de ce bill a donc décriminalisé les relations sexuelles entre deux adultes consentants de 21 ans et plus mais, dans le secteur public, les relations entre individus du même sexe sont demeurées sous le coup de la loi criminelle. Selon Kinsman (1996, p. 264), alors que les homosexuels préconisaient un débat public susceptible d'éduquer le public sur l'homosexualité et appuyer leur quête de légitimité et de respectabilité, le projet de loi a circonscrit les discussions autour de la régulation sexuelle en privé et en public et autour de l'implication des mineurs.

Dans les années qui ont suivi, le mouvement des gais et lesbiennes a poursuivi sa lutte. En 1971, une coalition canadienne de regroupements pour la libération des gais et des lesbiennes dénonce publiquement le peu d'impact de la réforme juridique sur l'oppression qu'ils subissent. Leur vie quotidienne est encore et toujours marquée par la discrimination, le harcèlement policier et des pressions pour rentrer dans le rang (Kinsman, 1996, p. 278). Au fil des années, les luttes apportent un certain nombre de changements. D'abord, le système médical et psychiatrique abandonne peu à peu la catégorisation de l'homosexualité comme maladie mentale ${ }^{9}$. On note l'émergence d'un marché de consommation homosexuel et d'une image positive des gais et lesbiennes. Les homosexuels se regroupent pour exiger la reconnaissance de leurs droits civiques : ils revendiquent l'insertion de l'orientation sexuelle comme motif de discrimination dans les Chartes canadienne et provinciales des droits de la personne aussi bien que l'accès aux bénéfices de conjoints dans les fonctions publiques provinciales et fédérales.

Mais à travers tous ces changements, on note l'émergence d'une distinction entre les «bons» homosexuels, qui sont «responsables» et recherchent la respectabilité, et les «mauvais» homosexuels, «irresponsables», qui sont encore étiquetés de déviants tant

9. Attention, ces changements ne constituent pas des renversements complets. En 1973, par exemple, l'American Psychiatric Association retire l'homosexualité de sa liste des troubles mentaux, mais la classe comme «trouble de l'orientation sexuelle». Cette association maintient la psychiatrisation des homosexuels non satisfaits de leurs conditions (Kinsman, 1996 : 290). Et ce n'est qu'en 1991 que l'Organisation mondiale de la Santé élimine l'homosexualité de son index international des maladies. 
au niveau social que sexuel. Cette homosexualité respectable ou du moins presque respectable renvoie à ceux dont la vie sexuelle apparaît structurée sur le modèle d'une relation affective stable à l'image de celle des couples hétérosexuels "respectables» (Kinsman, 1996, p. 358). Les «autres», dont le style de vie témoigne de la diversité des pratiques sexuelles, sont toujours confrontés à l'identité perverse homosexuelle. Par ailleurs, dans les années 1980, le mouvement politique queer qui émerge conteste ce redéploiement de la régulation sexuelle et des identités sexuelles. Sans prétendre rendre compte de la complexité du mouvement queer, notons que selon certains théoriciens, il exprimerait le refus d'une identité stable socialement acceptée "dont la légitimité repose essentiellement sur sa capacité de conférer une cohérence subjective à l'ensemble des personnes qui partagent une certaine orientation sexuelle» (Schwartzwald, 1998, p. 164). Ses partisans mettent l'accent sur la pluralité des pratiques sexuelles et sur la fluidité des identités sexuelles.

On constate alors que si les luttes des homosexuels ont permis de créer un certain espace social légitime pour plusieurs d'entre eux (espace relatif et fort fragile par ailleurs), et d'échapper à l'identité de l'homosexuel pervers, elles n'ont pas réussi à mettre en cause l'association entre sexualité et identité personnelle. En effet, les individus sont encore et toujours catégorisés à partir de leur orientation sexuelle et de certaines pratiques sexuelles; en défiant la sexualité normative hétérosexuelle et homosexuelle, ils se voient encore et toujours attribuer le statut de déviants.

\section{Et la lutte des travailleuses dans le secteur des services sexuels?}

La lutte pour permettre aux femmes qui vendent des services sexuels d'échapper à l'exclusion juridique et sociale n'a pas emprunté les mêmes voies et les résultats n'ont pas été aussi positifs.

D’abord, rappelons que les activités de prostitution ne bénéficieront pas d'un desserrement juridique dans les années 1960 au Canada et en Angleterre malgré le fait que l'influent Comité Wolfenden ait utilisé le même cadre d'analyse pour étudier les pratiques homosexuelles et les activités de prostitution. Même si les relations sexuelles proprement dites n'ont pas lieu en public, la catégorisation privé-public incite les législateurs à criminaliser la prostitution de rue au nom du «citoyen décent normal» offensé par la simple présence des prostituées dans les lieux publics. En 1959, le Parlement britannique adopte le Street Offence Acts alors qu'en 1972, le Canada définit la sollicitation publique pour fins de prostitution comme une infraction. La prostituée devra se soustraire au regard public ${ }^{10}$.

Malgré cet étau juridique, les prostituées emboîtent le pas au mouvement de libération des années 1960 et dans les années 1970 et 1980, des travailleuses de l'industrie du sexe de différents pays dont l'Angleterre, le Canada, les États-Unis, la France, la Hollande, etc., (Pheterson, 1989, 1996) prennent la parole. Elles rejettent la morale

10. Rappelons qu'au Canada, des infractions interdisent déjà formellement la prostitution dans des établissements et que sur la place publique, la prostituée pouvait être contrôlée à partir de la loi sur le vagabondage. La nouvelle loi accroît tout simplement les outils de répression. 
traditionnelle sur la sexualité et les analyses féministes dominantes à leur propos; elles affirment qu'elles ne sont ni des femmes dépravées dangereuses pour la société, ni des victimes exemplaires de l'ordre patriarcal, mais bien des travailleuses de l'industrie du sexe. Pour elles, la prostitution constitue un travail légitime et n'est pas plus associée à la contrainte, à l'esclavage que n'importe quel autre métier ouvert aux femmes dans nos sociétés patriarcales. Qui plus est, contrairement aux mythes véhiculés sur elles, les prostituées ne sont pas responsables de la propagation des maladies vénériennes; comme spécialistes dans leur domaine de travail, elles sont en mesure d'offrir des services sexuels sécuritaires. Elles réclament la décriminalisation de toutes les activités de prostitution et la reconnaissance de tous leurs droits civiques comme des travailleuses et citoyennes à part entière.

Notons ici que les demandes des regroupements de prostituées se distinguent de celles des groupes de femmes et des homosexuels en ce qu'elles ne réclament pas que ces pratiques sexuelles soient légitimées comme expression de leur sexualité; elles affirment plutôt qu'elles renvoient à des services sexuels et constituent une forme légitime de travail. Leurs revendications n'en impliquent pas moins un redéploiement de la régulation sexuelle : $y$ répondre favorablement, c'est reconnaître que des activités sexuelles puissent être investies de significations différentes selon le contexte social et individuel et qu'elles n'engagent pas nécessairement l'intimité d'un être. C'est mettre en cause l'association entre activités sexuelles et identité personnelle : leurs revendications touchent donc au cœur même de la sciencia sexualis, à l'idée que nos pratiques sexuelles définissent notre sexualité comme expérience subjective profonde et livrent la vérité de notre être. Pour les représentantes des regroupements de prostituées, ces activités qui sont des activités de travail, livrent simplement leur identité de professionnelles. Qui plus est, ces pratiques ne renvoient pas en elles-mêmes à l'asservissement des femmes par les hommes; elles peuvent aussi exprimer un rapport de services entre femmes et hommes. De cette façon, la sexualité n'est plus seulement conçue comme révélatrice de l'identité personnelle de l'individu, mais comme une question qui peut aussi être envisagée, apprise et pratiquée dans le cadre de la division sociale du travail.

Mais si on considère la lutte entreprise par les regroupements de prostituées après plus d'une décennie, il faut bien reconnaitre que leurs gains ont été plutôt modestes bien que tous les analystes ne soient pas unanimes quant au bilan à en tirer et aux explications à fournir.

McLeod (1982) et Jenness $(1993,1991)$ qui ont analysé le mouvement de soutien aux prostituées dans leur pays respectif en ont tiré un bilan positif. McLeod (1982) affirme qu'en Angleterre, le mouvement a permis aux prostituées de se faire entendre sur la place publique et a contribué à améliorer leur traitement aux mains des autorités en place. Jenness (1993, 1991), de son côté, a étudié l'impact de cоуоте (Call Of Your Old Tired Ethics), une organisation américaine vouée à la défense des droits des prostituées qui a vu le jour dans les années 1970. Elle affirme que le mouvement a réussi à imposer un discours alternatif sur la prostitution : à la définition de la prostitution comme péché et crime, on oppose maintenant celle de travail et de choix. Comme 
indice de changement au niveau de l'évaluation morale des activités de prostitution, Jenness invoque la percée du discours des regroupements des prostituées dans l'arène politique. Les femmes qui vendent des services sexuels s'affirment maintenant comme sujets politiques, mettent en cause les définitions dominantes de la prostitution comme problème social et proposent une réévaluation publique de la question. Elles se posent de plus en plus comme l'autorité légitime en matière d'analyse sur la prostitution, tirant le tapis sous les pieds des experts, des forces policières, des féministes traditionnelles et des professionnels de la santé. Le changement au niveau de la terminologie en témoigne : on parle maintenant de "travail dans le domaine des services sexuels», de «travailleuses du sexe». L'opposition que le mouvement a suscité démontre également que son impact n'est pas négligeable ${ }^{11}$. Il reste à voir, toujours selon Jenness, si ces changements seront institutionnalisés.

Par contre, tant Pheterson (1989), Weitzer (1991) que Poel (1995) penchent plutôt pour un verdict négatif. Dès 1989, devant la panique déclenchée par le sida, Pheterson (1989, p. 28) évoque le danger d'échec du mouvement de défense des prostituées en Europe comme en Amérique. Deux ans plus tard, Weitzer (1991), qui tout comme Jenness a étudié соуоте, conclut à l'échec du mouvement aux États-Unis. Il reconnaît que le mouvement a mis sur pied des services pour les prostituées, mobilisé les média, obtenu certaines concessions des autorités municipales et des corps policiers, gagné quelques batailles juridiques ponctuelles et a réussi à améliorer l'image de soi des prostituées impliquées dans le mouvement. Mais malgré ces quelques gains, il constate que le mouvement américain est toujours considéré comme une «croisade immorale» ou encore une cause qui n'offre pas d'avantages politiques et qui risque de se retourner contre ses promoteurs (Weitzer, 1991, p. 32). Poel constate également que malgré de nombreux appuis, le mouvement des prostituées n’a pas réussi à légitimer leur travail en Hollande.

En effet, on constate que même si le mouvement au États-Unis et ailleurs en Occident a introduit un discours alternatif autour de la définition du problème et assuré la visibilité de certaines représentantes des prostituées, il n'a pas réussi à orchestrer des changements concrets au niveau de l'exclusion juridique de ces activités, ni de renversement significatif au niveau de la condamnation morale dominante qui les accompagne. Ni le discours des prostituées ni l'échec séculaire des tentatives de contrôle pénal des activités de prostitution n'ont réussi à modifier l'attente normative face au commerce des services sexuels. La double image de la prostituée, dépeinte tantôt comme une femme perdue, dépravée, tantôt comme une jeune fille séduite, abandonnée ou écrasée par la misère, demeure solidement associée à la prostitution, imperméable aux faits bruts comme au discours politique.

11. Jenness fait état de la création de whisper (Women Hurt in Systems of Prostitution Engaged in Revolt) au début des années 1980 aux États-Unis. Ce mouvement, qui a émergé en opposition à coYote, s'est donné pour objectif non seulement de promouvoir la décriminalisation des activités de prostitution mais aussi de lutter pour son élimination. 


\section{COMMENT EXPLIQUER CES RÉSULTATS ?}

D'abord précisons que la définition du problème par les représentantes des prostituées s'est heurtée à la conception dominante de la prostitution dans les rangs féministes. Ces dernières n'ont offert au mieux qu'un appui mitigé et par ailleurs paradoxal aux revendications du mouvement des prostituées.

Dès les premiers moments du mouvement de libération des femmes, en effet, les féministes ont défini la prostitution comme le symbole de l'oppression des femmes, la forme ultime de l'appropriation de la sexualité des femmes par les hommes (Parent, 1994) ${ }^{12}$. Selon Kate Millet (1971/72), par exemple, la prostitution est au cœur de la condition féminine. Ces pratiques ne renvoient pas seulement à la déclaration publique de la soumission des femmes qui se concrétise de toutes façons dans le privé à travers le mariage, mais témoigneraient de la valeur des femmes, de notre dégradation, du pouvoir masculin ultime sur les femmes. «Ce n'est pas le sexe que la prostituée est amenée réellement à vendre : c'est de la dégradation. Et l'acheteur, le Jules, n'achète pas la sexualité mais le pouvoir, le pouvoir sur un autre être humain...» (Millet, 1971/72, p. 88). En somme, la prostitution constitue une forme d'esclavage, la réduction des femmes au statut d'objet sexuel, l'antithèse de l'être, de l'individu lui-même. Victime, elle ne peut se dégager de sa condition sociale qu'avec la libération des femmes.

Cette conception de la prostitution qui ignore la pluralité des contextes, des enjeux et des significations subjectives qui marquent les pratiques de prostitution, met l'accent sur la dimension de victimation et renvoie essentiellement aux différentes formes d'exploitation des femmes dans le cadre du commerce des services sexuels. Non seulement differe-t-elle de la conception de la prostitution au sein du mouvement des prostituées, mais elle appelle à la condamnation de toutes les pratiques de prostitution au nom de la morale civique et reconduit du même coup le jugement moral traditionnel sur la sexualité. Elle revêt même une forte dimension émotive lorsqu'elle s'appuie sur le témoignage de prostituées victimes de contraintes, de violences : l'expérience décrite est horrible, insoutenable, scandaleuse et la nécessité d'intervenir pour mettre fin à l'intolérable s'impose. Toute réflexion qui met en cause ces représentations peut difficilement trouver une oreille attentive dans les rangs féministes; faire entendre sa voix, c'est risquer d'être désignée comme irresponsable et, pire encore, d'être propulsée dans le camp ennemi, comme victime de fausse conscience et de ce fait, complice des exploiteurs ${ }^{13}$.

En réponse, les représentantes des regroupements de prostituées reconnaissent bien la présence de contrainte, de violence dans certains contextes de prostitution mais s'attardent peu à discuter de cette dimension. Il serait pourtant important qu'elles s'y engagent non pas pour discréditer le travail dans le domaine des services sexuels mais pour en tracer les frontières comme travail légitime. Nous sommes entièrement d'accord avec Rubin (1992, p. 302) lorsqu'elle affirme : «nous avons besoin d'analyser et de

12. La position féministe sur la question de la prostitution n'en est pas moins plurielle et fait l'objet de nombreux débats. À ce sujet, voir entre autres, Coderre et Parent (2001).

13. Nous avons repris ici certaines idées de Smart (1992) sur la controverse féministe en regard de la pornographie et nous l'appliquons à la prostitution. 
s'opposer aux situations d'inégalités qui marquent les rapports sociaux de sexe dans l'industrie du sexe. Mais ce n'est pas la même chose que d'essayer d'éliminer le commerce des services sexuels $»^{14}$. Par ailleurs, les témoignages des prostituées comme travailleuses dans l'industrie du sexe ne semblent pas avoir d'impact sur les représentations des féministes : tout se passe comme si la prostitution ne pouvait comprendre comme phénomène qu'un type d'expériences, qu'un type de définitions de ces expériences. Les représentantes des prostituées prennent encore une fois figure de femmes rebelles, exceptionnelles, qui ne manifestent aucune empathie face à la victimation de leurs sœurs ou encore face à la situation globale d'oppression des femmes par les hommes. Cette lutte autour de la définition du problème affaiblit le mouvement des prostituées.

Cette dissension au niveau de la conception du problème, on le comprend, a largement orienté les relations entre les féministes et les regroupements des prostituées. Les féministes radicales ont bien voulu, malgré les différences, donner leur appui aux prostituées et ont recommandé la décriminalisation des activités de prostitution (Parent, 1994). Plusieurs d'entre elles, dont Millet (1971/72), spécifient d'ailleurs que ce sont les prostituées elles-mêmes qui sont les mieux informées et les plus aptes à diriger la lutte face aux lois contre la prostitution et à demander la reconnaissance des droits juridiques pour les prostituées. Mais pour les féministes radicales, la prostitution doit être abolie : il n'est pas question de lutter pour la reconnaissance juridique et sociale de ces activités. Aussi ne faut-il pas s'étonner que la prostitution constitue une problématique fort controversée dans les rangs féministes et maints analystes ont constaté le retard de l'appui féministe dans la cause menée par les regroupements de prostituées (Jaget, 1980; Jenness, 1990; Weitzer, 1991), voire même dans la défense de dossiers juridiques impliquant des prostituées et la question de la prostitution (Bell, 1987; McGinnis, 1994). L'appui concret mitigé et paradoxal des féministes constitue à son tour un frein pour le mouvement des prostituées.

Les représentantes des regroupements de prostituées doivent également lutter contre le discrédit qui entoure les activités de prostitution. Les lois bien sûr contribuent à maintenir les prostituées dans l'ombre : toute affirmation publique de ce «métier déviant» peut se solder en arrestation ou encore en harcèlement policier. D’autant plus qu'on associe encore la petite prostitution avec le monde du crime organisé ou du moins avec le monde des drogues illégales, voire avec celui de l'exploitation des enfants. La stigmatisation sociale des prostituées constitue une autre menace : dévoiler ses activités peut vouloir dire être évincée de son logement, subir le harcèlement des voisins, perdre la garde de ses enfants, etc. (Pheterson, 1989). Sortir de l'ombre est donc potentiellement lourd de conséquences et on comprend que les regroupements de prostituées ne puissent compter sur un nombre important de membres officielles et de sympathisantes ${ }^{15}$ et ne disposent que de peu de ressources matérielles et organisation-

14. Nous avons traité de cette question dans une publication antérieure. Voir Parent (1994).

15. Mcleod (1981, p. 71)) affirme que les femmes qui ont rejoint l'organisation Pros en Angleterre ont dû faire face à de nombreuses difficultés; Poel (1995. p. 47) constate à son tour, qu'en Hollande, les prostituées préféraient demeurer dans l'anonymat plutôt que de joindre un regroupement de prostituées. 
nelles pour leur lutte. Selon Weitzer (1991), le mouvement des prostituées aux États-Unis ne renvoie pas à un mouvement de base mais plutôt à un mouvement professionnel où les leaders parlent au nom des prostituées mais sans que ces dernières ne soient directement impliquées ni vraiment mobilisées pour apporter leur appui. C'est d'ailleurs ce qui l'a amené à conclure à l'échec du mouvement соуоте. Selon lui, si le mouvement des prostituées avait pu compter sur un support substantiel de ses membres ou encore d'un groupe de sympathisants influents, il aurait pu enregistrer un succès comparable à celui du mouvement des homosexuels qui a pu prendre appui sur une culture gaie et lesbienne, de meilleures sources de financement et des organisateurs chevronnés. Les facteurs moraux et idéologiques lui apparaissent secondaires pour expliquer l'échec du mouvement.

Mais cette analyse ne fait pas l'unanimité et nous croyons, quant à nous, qu'elle fait l'économie de la complexité des enjeux. Poel (1995) qui a étudié la lutte des prostituées en Hollande conteste non pas le diagnostic de Weitzer (1991) mais plutôt son analyse des facteurs responsables de l'échec. Elle constate qu'en Hollande, le mouvement des prostituées, le Red Thread, a bénéficié de ressources financières, organisationnelles aussi bien que d'appuis politiques mais n'a pas davantage réussi à faire reconnaitre une prostitution professionnelle légitime en Hollande ${ }^{16}$. La lutte menée s'est tout simplement soldée par un élargissement du filet, par une extension du contrôle social de la prostitution. Pourtant, les objectifs du mouvement étaient clairs et axés sur l'autonomie des prostituées : on voulait abroger l'article de loi qui interdisait la tenue de maisons de prostitution et on préconisait la formation d'une coopérative de prostituées pour celles qui souhaitaient exploiter elles-mêmes une maison de prostitution de petite taille. Le gouvernement devait apporter son appui financier à cette initiative et fournir des renseignements sur la gestion de petite entreprise. Il devait également prévoir des mesures d'appui ${ }^{17}$ pour les prostituées qui voulaient quitter le commerce. Ces recommandations émanaient des efforts concertés de représentantes du mouvement des femmes, de politiciens, de professionnels de l'État. Comment Poel (1995) en explique-t-elle l'échec malgré cette belle unanimité?

Tant que le mouvement a mis l'accent sur la prostitution professionnelle comme commerce usuel et régulier, affirme-t-elle, il a bénéficié d'un appui certain. Mais très tôt, on a voulu intégrer les prostituées-hérö̈nomanes ${ }^{18}$ parmi les prostituées professionnelles; ce faisant, on a mis l'accent sur les situations problématiques, sur les dimensions de violence et de contrainte dans la prostitution et plutôt que de renouveler le regard sur les prostituées, on a exarcerbé la stigmatisation traditionnelle à leur égard. On est ainsi passé d'une lutte pour la reconnaissance d'un métier à la mise en place de

16. Le mouvement a reçu l'appui de professionnels responsables des politiques au sein du gouvernement, de parlementaires, des média, de la police, des gouvernements locaux et national.

17. Le gouvernement devait s'engager à fournir un logement en dehors des secteurs de prostitution, à effacer les dettes qu'elles avaient contractées et qu'elles ne pourraient rembourser si elles quittaient le métier. 18. Des travailleurs sociaux ont souligné la nécessité d'incorporer cette catégorie de prostituées; on a également voulu intégrer la prostitution juvénile et le tourisme sexuel. 
services individuels pour les prostituées dans le besoin. Le mouvement homosexuel, souligne Poel (1995), n'avait pas commis une telle erreur; les organisateurs avaient bien pris soin de cibler une homosexualité acceptable pour fin de reconnaissance et de reléguer dans l'ombre les éléments déviants du groupe. Il faut dire que le sida a joué un rôle de catalyseur dans la réorientation de la lutte des regroupements des prostituées en Hollande : là comme ailleurs en Occident, le spectre de la maladie a suscité un climat de panique, de retraite vers des formes plus traditionnelles de comportements sexuels (Brock, 1989; Weeks, 1993b, 1995) et a ravivé l'association traditionnelle entre les prostituées et la propagation de maladies vénériennes malgré les recherches qui infirment cette croyance (Haug et Nicci, 1984).

Pour Poel (1995, p. 63), la solidarité avec toutes les catégories de prostituées s'est retournée contre le mouvement :

La solidarité est une noble ambition, mais on doit être assez fort pour se le permettre. L'exemple de la Hollande nous enseigne que l'intégration des catégories de prostituées qui correspondent le plus aux stéréotypes traditionnels sur la prostitution et sur les prostituées ne profite pas à toutes les catégories mais entraine une dépréciation du groupe entier.

Weitzer et Poel renvoient donc à des facteurs différents pour expliquer l'échec du mouvement des prostituées dans leur pays respectif, soit les États-Unis et la Hollande. Pour Weitzer (1991), l'échec du mouvement est attribuable au manque de ressources. Pour Poel (1995), l'échec repose plutôt sur une mauvaise stratégie dans la construction de l'image de la prostituée professionnelle. Les deux ont assurément cerné des points névralgiques de la lutte des prostituées : aux États-Unis, COYOTE n'a pas été créé suite à la formation d'un réseau culturel susceptible de constituer un appui pour les femmes œuvrant dans l'industrie du sexe; en Hollande, l'image de la prostituée professionnelle a été associée très tôt à l'image traditionnelle de la prostituée victime, en danger et dangereuse. Cette grille de lecture pour comprendre le succès du mouvement homosexuel et l'échec du mouvement des prostituées à partir de leur lutte pour leur intégration sociale est éclairante. Mais nous pouvons également considérer les résultats de ces mouvements à partir des changements qui marquent la modernité en ce qui concerne la construction de la sexualité. Giddens (1992, p. 32) nous propose à cet effet une seconde grille de lecture, non pas, dit-il, pour infirmer la première, mais pour situer les enjeux de ces luttes dans le cadre plus large des changements qui marquent la modernité. La socialisation du monde naturel accompagne ces changements, affirme-t-il, et plus particulièrement la socialisation de la reproduction, autrefois fermement ancrée dans la nature. Ce faisant, l'hétérosexualité n'apparait plus comme la norme absolue et l'on assiste à un déclin des perversions. La sexualité devient enjeu de plaisir et d'expérimentation (Vance, 1984). Ces changements ouvrent un espace pour la diversité sexuelle mais faut-il encore organiser la lutte pour concrétiser et maintenir ces changements.

Mais si cette lecture proposée par Giddens (1992) renvoie à des enjeux plus larges qui nous apparaissent pertinents pour saisir certaines tendances, elle ne nous permet pas de rendre compte de l'ensemble des changements au niveau de la régulation sexuelle et du maintien de l'exclusion de la prostitution. D'abord, le déclin des perversions n'est 
pas généralisée : si certaines s'effacent, d'autres prennent maintenant l'avant-scène, et nous pensons à la pédophilie, aux pratiques sado-masochistes, etc. Notons que les pratiques particulièrement dénoncées aujourd'hui comme perverses renvoient à des comportements sexuels marquées par des relations de pouvoir fortement inégalitaires, par la contrainte, voire par la violence. Si certains démons entrent dans l'ombre, c'est pour faire place à d'autres. À travers tous ces changements, la prostitution demeure largement stigmatisée : les lois contribuent encore et toujours à exclure ces pratiques, on cherche encore l'explication du choix des prostituées dans leurs déficits psychologiques et sociaux et l'on maintient leur exclusion sociale. Comment comprendre une telle résistance aux changements?

D'abord, comme nous l'avons vu, le mouvement des prostituées présente la prostitution comme un travail et dissocie le lien savant et culturel entre identité personnelle et sexualité. À ce titre, ses représentantes défendent une position qui demeure fortement subversive dans le nouveau paysage de la régulation sexuelle qui a émergé depuis les années 1960. Ce moment marque, bien sûr, la fin des discours hégémoniques (religieux, scientifiques) qui contrôlaient les comportements sexuels pour faire place à un monde pluraliste : aujourd'hui, la question des comportements sexuels, de l'identité sexuelle et des mœurs sexuelles renvoient plutôt à des choix individuels (Weeks, 1995, p. 27). Giddens (1992) suggère d'ailleurs qu'en cette période postmoderne, le questionnement qui s'impose est «comment dois-je vivre ma vie»? Cette question se pose particulièrement dans la sphère de l'intimité, en ce qui concerne les relations domestiques et la sexualité. Devant la pluralité d'options possibles, on s'interroge sur les bons choix et on recherche une relation satisfaisante comme moyen d'affirmation de soi. On s'engage alors non plus dans un contrat de mariage à vie, mais dans une relation intime qui devra être bâtie au quotidien, qui demande l'implication démocratique des partenaires, et qui, contrairement au mariage traditionnel, loge sous le signe de la flexibilité mais aussi de l'incertitude. La relation qui se construit alors constitue, selon Giddens, l'élément fondamental de la sphère de l'intimité ${ }^{19}$. Qui plus est, le développement de cette relation dans le cadre du quotidien intime fournit à l'individu une histoire de vie qui lui permet de se reconnaître une identité personnelle, toute provisoire soit-elle, dans ce monde marqué par l'incertitude. La sexualité fait corps avec cette sphère de l'intimité et avec la formation de l'identité individuelle.

Devant ces changements, nous croyons que nous faisons face à un obstacle majeur au niveau de la conception de la prostitution. On peut voir comment sa conception comme travail ajoute à l'insécurité qui marque les choix individuels d'aujourd'hui. Les gestes sexuels sont encore et toujours associés à la sexualité de la personne, à la formation de son être intime; c'est à partir, entre autres, de ce présupposé, qu’on essaie de

19. Giddens (1992, p. 130) reconnaît que les femmes ont cheminé davantage que les hommes dans cette voie jusqu'à maintenant et que les homosexuels sont mieux placés que les hétérosexuels pour suivre ce chemin. Aux hommes hétérosexuels d'accepter maintenant d'investir leur identité dans une relation affective marquée non plus du signe de leur autorité mais de la marque de partenaires égaux qui «grandissent» ensemble. 
se construire comme individu dans un monde où les repères font cruellement défaut. Accepter de dissocier chez la même personne gestes sexuels professionnels et expérience sexuelle intime, c'est secouer ce qu'on considère comme un repère de la formation de l'identité personnelle et non pas simplement accepter la pluralité des identités sexuelles, ce qui constitue d'ailleurs déjà un défi ${ }^{20}$.

Enfin, en cette époque où les perversions qu'on dénonce impliquent inégalité de pouvoir, contrainte, voire violence, la prostitution cadre d'entrée de jeu avec l'insoutenable. Les prostituées peuvent apparaître comme des victimes à double titre : à cause des violences physiques occasionnelles subies dans le cadre de leurs pratiques mais aussi à cause du mépris ou encore de la réification dont elles peuvent être l'objet et qui contraste avec le développement des relations hommes-femmes sous le signe d'une plus grande égalité. Les féministes elles-mêmes définissent les prostituées comme les victimes ultimes de l'oppression des hommes. On a du mal à concevoir qu'elles peuvent se définir comme des professionnelles qui contrôlent les services qu'elles offrent : on les voit comme des femmes dont le moi intime est mis au service de l'autorité sexuelle des hommes.

Compte tenu du contexte social et cognitif, on comprend bien que le soutien au mouvement des prostituées soit plutôt limité et que leur initiative puisse rapidement être discréditée. Leur lutte ébranle très fortement nos représentations de la sexualité et de la victimation des femmes.

Les quelques victoires que le mouvement des prostituées a pu enregistrer (attention des média, certains appuis policiers, de représentants municipaux, de professionnels, d'intellectuels, certaines batailles juridiques) constituent peut-être non seulement un gain symbolique mais déjà un tour de force. Mais il semble bien que la légitimité de leurs pratiques ne pourra être acquise que lorsqu'on acceptera d'établir une moralité non pas à partir des pratiques sexuelles mais de la responsabilité des acteurs sociaux face aux relations impliquées.

\section{RÉSUMÉ}

Pourquoi les luttes menées par les regroupements de prostituées depuis les années 1970 n'ontelles pas réussi à transformer les attentes normatives face à la prostitution? Comment expliquer que les femmes et les gais et lesbiennes aient réussi à introduire certaines conditions de possibilité de changements au niveau de leur identité sexuelle et non pas les prostituées? Nombre de facteurs semblent avoir joué un rôle mais l'articulation entre sexualité, intimité, vie conjugale et identité personnelle apparaît compromettre fortement les possibilités de faire valoir une conception de la prostitution comme travail.

20. Notons que l'émergence du sida nous a plongé dans une économie sexuelle de récession, où sexualité se conjugue à nouveau avec peur, avec danger (Weeks, 1993b, 1995). Nous pouvons bien comprendre que dans ce climat de peur épidémique, le vieux spectre de la prostitution comme véhicule de la mort reprend vite droit de cité et que les prostituées deviennent les boucs émissaires. On veut les exclure ou on veut les sauver, mais on ne saurait accepter leurs pratiques. 


\section{SUM MARY}

Why have struggles by groups of prostitutes since the 1970s not succeeded in transforming normative expectations in regard to prostitution? How can we explain that women and gays and lesbians have succeeded in introducing certain conditions for the possibility of change on the level of sexual identity and not prostitutes? A number of factors seem to have played a role, but the link between sexuality, intimacy, conjugal life and personal identity seem to compromise seriously any possibility of asserting a conception of prostitution as work.

\section{RESUMEN}

¿Por qué las luchas llevadas a cabo por los grupos de prostitutas a partir de la década de 1970 no llegaron a transformar las expectativas normativas frente a la prostitución? ¿Cómo explicar que las mujeres y los gays y lesbianas hayan llegado a introducir ciertas condiciones de posibilidad de cambios en cuanto a su identidad sexual y no las prostitutas? Numerosos factores parecen haber jugado un rol, pero la articulación entre sexualidad, intimidad, vida conyugal y identidad personal parece comprometer de manera importante las posibilidades para hacer valer una concepción de la prostitución como trabajo.

\section{BIBLIOGRAPHIE}

Bell, L. (éd.) (1987), Good Girls/Bad Girls. Feminist and Sex Trade Workers Face to Face, Toronto, The Women's Press.

Bell, S. (1994), Reading, Writing \& Rewriting the Prostitute Body, Bloomington, Indiana University Press. Brock, D. (1998), Making Work, Making Trouble. Prostitution as a Social Problem, Toronto, Toronto University Press.

BRock, D. (1989), «Prostitutes are Scapegoats in the AIDs Panic», Resources for Feminist Research, vol. 18, $\mathrm{n}^{\circ}$ 2, p. 13-17.

Cellard, A. et G. Pelletier (1998), «Le Code criminel canadien, 1892-1927 : Étude des acteurs sociaux», The Canadian Historical Review, vol. 79, $\mathrm{n}^{\circ}$ 2, p. 261-303.

Coderre, C.et C.Parent (2001), «Le Deuxième Sexe et la prostitution. Pour repenser la problématique dans une perspective féministe», in C. Coderre et M.B. Tahon (éd.), Le Deuxième Sexe. Une relecture en trois temps, 1949-1971-1999, Montréal, Les Éditions du Remue-Ménage, p.73-89.

Chapkis, W. (1997), Live Sex Acts. Women Performing Erotic Labor, New York, Routledge.

Conseil Consultatif Canadien sur la situation de la femme (1984), La Prostitution au Canada, Ottawa.

Demers, R. (1984), «De la lex scantinia aux récents amendements du Code criminel : homosexualité et droit dans une perspective historique», Les Cahiers du Droit, vol. 25, p. 777-800.

Descarries-Bélanger, F. et S. Roy (1988), Le mouvement des femmes et ses courants de pensée : essai de typologie, Ottawa, ICREF.

Dubois, E.C. et L. Gordon (1983), «Seeking Ecstasy on the Battlefield: Danger and Pleasure in Nineteenth Century Feminist Sexual Thought», Feminist Studies, vol. 9, n 1, p. 7-25.

Foucault, M. (1980), «La poussière et les nuages», in M. Ревrot (éd.), L'impossible prison. Recherches sur le système pénitentiaire au XIX ${ }^{e}$ siècle, Paris, Éditions du Seuil, p. 9-56.

Foucault, M. (1976), Histoire de la sexualité, 1, La volonté de savoir, Paris, Gallimard.

Giddens, A. (1992), The Transformation of Intimacy. Sexuality, Love \& Eroticism in Modern Societies, Stanford, Cal., Stanford University Press.

HaUg, M. et M. Nicci (1984), "Prostitution et propagation des maladies transmises sexuellement», Documents de Travail sur la pornographie et la prostitution, rapport $n^{\circ} 7$, Ottawa, Ministère de la Justice. Jaget, C. (éd.) (1980), Prostitutes, Our Life, Bristol, Angl., Falling Wall Press. 
JAGgar, A. (1983), Feminist Politics and Human Nature, Totowa, Rowman and Allanheld.

Jenness, V. (1993), Making it Work. The Prostitutes'Rights Movement in Perspective, New York, Adline de Gruyter.

Jenness, V. (1990), «From Sex as Sin to Sex as Work: coyote and the Reorganization of Prostitution as a Social Problem», Social Problems, vol. 37, nº 3, p. 403-420.

Kinsman, G. (1996), The Regulation of Desire. Homo and Hetero Sexualities, Montréal, Black Rose Books.

McGinnis, J.D. (1994), «Whores and Worthies: Feminism and Prostitution», Revue canadienne de droit et société, vol. $9, \mathrm{n}^{\circ} 1$, p. 105-121.

McLeod, E. (1982), Women Working: Prostitution Now, London, Croom Helm.

Mcleod, E. (1981), «Man-Made Laws For Men? The Street Prostitutes' Campaign Against Contro », in B. Hutter, G. Williams (éd.), Controlling Women. The Normal and the Deviant, Londres, Croom Helm London and the Oxford University Women's Studies Committee, p. 61-78.

Millet, K. (1971/1972), La Prostitution. Quatuor pour voix féminines, Paris, Denoël/Gonthier.

Parent, C. (1994), «La prostitution ou le commerce des services sexuels», in S. Langlois, Y. Martin et F. Dumont (éd.), Traité de problèmes sociaux, Québec, Institut québécois de recherche sur la culture, p. 393-407.

Parent, C. et C. Coderre (2000), «Le corps social de la prostituée : regards criminologiques», in S. Frigon et M. Kérésit (éd.), Du corps des femmes. Contrôles, surveillances, résistances, Ottawa, Les Presses de l'Université d'Ottawa, p. 93-124.

Pheterson, G. (1996), The Prostitution Prism, Amsterdam, Amsterdam University Press.

Pheterson, G. (1989), A Vindication of the Rights of Whores, Seattle, wa, Seal Press.

Poel (VAn DER), S. (1995), «Solidarity as Boomerang, The Fiasco of the Prostitutes' Rights Movement in the Netherlands ", Crime, Law and Social Change, vol. 23, p. 41-65.

Richardson, D. (1993), «Sexuality and Male Dominance», in D. Richardson et V. Robinson (éd.), Thinking Feminist. Key Concepts in Women's Studies, New York, The Guilford Press, p. 74-98.

Rubin, G. (1992), «Thinking Sex: Notes for a Radical Theory of the Politics of Sexuality», in C. VAnce (éd.), Pleasure and Danger. Exploring Female Sexuality, Londres, Pandora Press, p. 267-319.

Schwartzwald, R. (1998), «Y a-t-il un sujet-nation queer? Apories d'un débat actuel», in D. Lamoureux (éd.), Les limites de l'identité sexuelle, Montréal, Les Éditions du Remue-Ménage, p. 163-179.

Schulz, M. (1998), «Lesbiennes : les silences du droit», Les Temps modernes, vol. 598, p.113-155.

Seidman, S. (1992), Embattled Eros. Sexual Politics and Ethics in Contemporary America, New York, Routledge. Shaver, F.M. (1994), "The Regulation of Prostitution: Avoiding the Morality Trap », Revue canadienne de droit et société, vol. $9, \mathrm{n}^{\circ}$ 1, p.123-145.

SMART, C. (1992), «Unquestionably a moral issue. Rhetorical devices and regulatory imperatives», in L. Segal et M. Mcintosh (éd.), Sex Exposed. Sexuality and the Pornography Debate, New Brunswick, New Jersey, Rutgers University Press, p. 184-199.

Tamagne, F. (1998), «Naissance du troisième sexe», L'histoire, vol. 221, p. 48-55.

Tong, R. (1989), Feminist Thought. A Comprehensive Introduction, Boulder, Westview Press.

Walkowitz, J.R. (1991), «Sexualités dangereuses», in G. Ruby et M. Рerrot (éd.), Histoires des femmes en Occident, Le XIX ${ }^{e}$ siècle, sous la direction de Geneviève Fraisse et Michelle Perrot, Paris, Plon, p. 389-418.

Walkowitz, J.R. (1980), Prostitution and Victorian Society, Cambridge, New York, Cambridge University Press.

WeEks, J. (1995), Invented Moralities. Sex Values in the Age of Uncertainty, New York, Columbia University Press.

Weeks, J. (1993a), «An Unifinished Revolution: Sexuality in the $20^{\text {th }}$ Century», in v. Hardwood et al (éd.), Pleasure Principles. Politics, Sexuality and Ethics, London, Lawrence \& Wishart, p. 1-19.

Weeks, J. (1993b), «Aids and the Regulation of Sexuality», in V. Berridge et P. Strong (éd.), AIDS and Contemporary History, Cambridge University Press, p. 17-36.

Weeks, J. (1981), Sex, Politics and Society: The Regulation of Sexuality since 1800, Harlow, Longman.

Weitzer, R. (1991), «Prostitutes' Rights in the United States: The Failure of a Movement», The Sociological Quaterly, vol. 32, $\mathrm{n}^{\mathrm{o}}$ 1, p. 23-41. 